\title{
Impact of Long-Term use of Mobile Phones on the Prostate in Human users
}

\author{
${ }^{1}$ Madyha Hassan Mahmoud, ${ }^{2}$ Nadia Youssef Morcos, ${ }^{3}$ Khadiga Salah Ibrahim, \\ ${ }^{3 *}$ Amal Saad-Hussein, ${ }^{1}$ Noha Hassan Ibrahim and ${ }^{2}$ Ahmed Fathi Soliman \\ ${ }^{1}$ Department of Clinical and Chemical Pathology, National Cancer Institute, Cairo University, Cairo, Egypt \\ ${ }^{2}$ Department of Biochemistry, Faculty of Science, Ain Shams University, Cairo, Egypt \\ ${ }^{3}$ Department of Environmental and Occupational Medicine, National Research Center, Giza, Egypt
}

\author{
Article history \\ Received: 27-09-2020 \\ Revised: 21-12-2020 \\ Accepted: 22-12-2020 \\ Corresponding Author: \\ Amal Saad-Hussein \\ Department of Environmental \\ and Occupational Medicine, \\ National Research Center, \\ Giza, Egypt \\ Email:amel_h@hotmail.com
}

\begin{abstract}
The current study investigated some possible effects of long-term use of mobile phones on the oxidative-antioxidants balance and its effect on the prostate. Twenty-eight eligible volunteers frequently use mobile phones and from the same socioeconomic status were enrolled in the study. They were examined according to the effect of time every two years for a follow-up period with four years. A detailed personal, occupational and medical questionnaire was completed for each participant through personal interviews. Total and free Prostate-Specific Antigen (PSA) levels, in addition to the levels of malondialdehyde, Total Antioxidant Capacity (TAC) and Zinc (Zn) were determined. At the end of the study, total and free PSA and MDA levels increased significantly compared to the baseline. Compared to the two years of use, total and free PSA level, free PSA/total PSA ratio, MDA and Zn levels were significantly increased after 4 years. In conclusion, the long-term use of cell phones may cause a redox imbalance characterized by increased oxidative stress that eventually affected the prostate gland.
\end{abstract}

Keywords: Mobile Phones, Prostate-Specific Antigen (PSA), Total Antioxidant Capacity (TAC), Zinc, Malondialdehyde (MDA)

\section{Introduction}

Many sources such as mobile phones, computers, power transmission lines, radars and electrical equipment are increasing the amount of human exposure to non-ionizing electromagnetic radiations (Parsanezhad et al., 2015). On talking into a mobile phone, the sound wave is transferred through a transmitter from the speaker. The transmitter converts the sound into a sine wave that operates at approximately 0.75 to 1 watt of power, with 2 watts at peak use and through the transmitter circuit the electrical sine wave current creates an Electromagnetic Field (EMF) in its region. As the electrical current moves back and forward, the fields continue to build up and collapse, creating electromagnetic radiation. Thus, the transmitter generates mobile phone radiation through the antenna; in the form of Radiofrequency Electromagnetic Waves (RF-EMW) (Agarwal et al., 2011). Modern advances in mobile phone telecommunication systems are related to an enhanced signal frequency, which correlates with higher energy radiofrequency waves (Dasdag and Akdag, 2016).

Reactive Oxygen Species (ROS) are continuously neutralized by antioxidants present in body tissues and whenever the production of ROS exceeds the scavenging capacity of antioxidants, it leads to Oxidative Stress (OS) (Desai et al., 2010). Over the last decade, in vivo animal studies have shown that OS was developed in response to mobile phone radiation due to the disturbance in ROS metabolism by increasing its production or decreasing the activity of antioxidant enzymes (Al-Damegh, 2012). Meena et al. (2014) reported that chronic exposure to RFEMW has been shown to reduce the activity of some antioxidants; such as catalase, Superoxide Dismutase (SOD) and Glutathione Peroxidase (GPx), which consequently decreases the total antioxidant capacity in different organs of the body. In another study, spermatozoa SOD was significantly reduced in ejaculated semen after exposure to RF-EMW radiation compared to the unexposed control. This is indicative that electromagnetic waves emitted from cellular phones can increase ROS formation causing a heightened oxidative stress to spermatozoa (Baah et al., 2019).

Prostate Cancer (PC) is a common health problem. It grows slowly, usually in the outer part of the gland, but, in advanced metastatic cases, it spreads beyond the prostate very quickly (Jemal et al., 2011). The oxidative 
stress/antioxidant imbalance status has been observed in prostate cancer patients (Donkena et al., 2010).

Therefore, this study aimed to explore the oxidative stress effect of long-term use of cell phones on the prostate.

\section{Subjects and Methods}

\section{Study Population and Design}

The present study was a prospective self-control study conducted over four years on apparently healthy males. The study was started with 50 mobile user males from the same socioeconomic status, but due to the very huge exclusions criteria and the long period of follow-up (4 years followup), the number of subjects who completed the study was decreased to 28 males. These 28 subjects had voluntarily completed the study to detect the effect of time every two years with a total follow-up period of four years.

At the beginning of the study, the participants aged between 19 and 45 years. Selection of the included subjects was in this young age to exclude the confounding factors that may develop prostate symptoms during the long period of follow-up (4 years):

- Inclusion criteria: Male used mobile phones for 5 years or more before beginning the study

- Exclusion criteria: Females had been excluded from the study. Males with high blood pressure, hepatitis $\mathrm{B}$ virus, or hepatitis $\mathrm{C}$ virus had also been excluded. Confounding factors were also almost controlled during the selection of the voluntary subjects included in the study, as subjects suspected to develop any confounding exposures had been excluded; such as smoking, aging and residential or occupational exposure to EMF

This study followed the Helsinki Declaration on human research. Informal consent was obtained from all participants included in the study.

\section{Questionnaire}

At the beginning of the study, a detailed personal, occupational and medical questionnaire was completed through personal interviews with each participant. It included questions about age, social status, mobile usage patterns (number and types of the mobile, frequency of daily use of phones, manner and location of the phone during sleeping). Symptoms of prostatic problems including the presence of weight loss, complaints of rushing to the toilet, difficulty in urination, or nocturnal urination were also included in the questionnaire.

\section{Blood Samples}

Five $\mathrm{ml}$ of venous blood were drawn from all the included subjects under septic conditions at the beginning of the study and after 2 and 4 years; as the same investigations were done to detect any changes during this long period of the study.

Each sample was divided into two parts; one $(3 \mathrm{~mL})$ was collected in dry tubes for serum biochemical analysis and the other $(2 \mathrm{~mL})$ was collected into Ethylene Diamine Tetra-acetic Acid (EDTA) tubes to obtain the plasma.

\section{Prostate Biomarkers}

Serum levels of both total and free Prostate-Specific Antigen (PSA) were quantitatively determined by the Elecsys $^{\circledR}$ immunoassay kits (Roche Diagnostics) based on an Electro-Chemiluminescence Immunoassay (ECLIA). The assay lower detection limit was 0.002 $\mathrm{ng} / \mathrm{mL}$ for total PSA and $0.01 \mathrm{ng} / \mathrm{mL}$ for free PSA with intra- and inter-assay Coefficient of Variation $(\mathrm{CV})<8$ and $<10 \%$, respectively for both assays. The test was performed on the Cobas e 411 analyzers (Roche Diagnostics, Risch-Rotkreuz, Switzerland). The ratio of free PSA/total PSA was then calculated.

\section{Oxidative Stress Parameters}

Malondialdehyde (MDA) was measured in plasma according to the method described by (Kei, 1978) using a commercially available kit (Biodiagnostic, Giza, Egypt).

Total Antioxidant Capacity (TAC) was estimated spectrophotometrically in serum according to the method of (Koracevic et al., 2001) using an available commercial kit purchased from Biodiagnostic (Giza, Egypt).

\section{Serum Zinc}

Serum zinc concentration was determined by colorimetric method according to (Johnsen and Eliasson, 1987) using an available commercial kit provided by Greiner Diagnostic GmbH (Germany) following the manufacturer's instructions.

\section{Statistical Analyses}

Distribution of quantitative data was tested using the Shapiro-Wilk test, normally distributed data was expressed as mean \pm SD and non-normally distributed data was expressed as the median and interquartile range (25th and 75th percentile). Qualitative data was represented by frequency and percent. Continuous variables were compared between each of the two groups using the paired t-test, or Wilcoxon signed ranks test as appropriate. Wilcoxon signed ranks test was also performed to detect the relative percent change. The probability ratio was used to compare qualitative data. Spearman's correlation analysis was used to assess the relationship between every two variables. P-values were 2sided and the P-value $<0.05$ was considered to be statistically significant. Statistical analysis was performed using SPSS version 24 (IBM Corp, NY, USA). 


\section{Results}

\section{Demographic Data and Mobile Use Habits of the Study Population}

The included males aged from 19 to 45 years at the beginning of the study and their baseline characteristics are shown in Table 1. At the beginning of the study, 12 participants were single and at the end of study five of them were married. All the participants kept their mobile phones in the pocket during the day from the start to the end of the study. None of the included subjects complained from blood in their urine.

Changes in mobile usage patterns after 2 and 4 years of the study as compared to the beginning are illustrated in Fig. 1. The relative percent change of use/year increased substantially after 2 and 4 years of use. The frequency of use/year increased significantly after the second and fourth year of use compared to the start of the study (baseline). However, the frequency of use/day was increased significantly after 2 years but decreased significantly after 4 years of use compared to the baseline of the study.

The habits of using the mobile and types of phones used during the 4 years of study are shown in Fig. 2. The habits studied included the number and types of phones, whether the mobile was on or off and the location of the phone during sleeping hours. The figure shows that $89 \%$ of the participants were using two types of phones at the end of the study compared to only $4 \%$ at the beginning of the study, but not to the level of significance (Likelihood ratio $=7.512, \mathrm{P}=$ 0.11 ). The habits of putting the mobile phones aside during sleeping did not change significantly over time (Likelihood ratio $=0.08, \mathrm{P}=0.96)$, as well as switching the mobile off during sleeping (Likelihood ratio $=0.049, \mathrm{P}=0.98$ ).

\section{Changes in the Symptoms During the 4 Years of the Study}

The symptoms of prostatic problems among the participants during the study period are shown in Fig. 3. The complaints from rushing to the toilet and weight loss were not changed during the study period (Likelihood ratio $=0.192, \mathrm{P}=0.908$; Likelihood ratio $=0.686, \mathrm{P}=$ 0.710 respectively). On the other hand, the percentage of the participants complaining about difficulty in urination increased to $40 \%$ at the end of the study, but without significant differences (Likelihood ratio = 3.559, $\mathrm{P}=0.169$ ). Moreover, no one complained of frequent night-time urination at the beginning, but 13 and $12 \%$ began to complain of night-time urination, without significant difference (Likelihood ratio $=$ 0.192, $\mathrm{P}=0.908$ ).

\section{Variation in the Biochemical Parameters}

Table 2 shows that compared to the beginning of the study, the participants had significantly higher levels of MDA after 2 years of using mobile phones, while, the levels of free PSA, free PSA/total PSA ratio, TAC and serum $\mathrm{Zn}$ were significantly reduced. After 4 years of phone usage, total PSA, free PSA and MDA levels and the free PSA/total PSA ratio were significantly increased, while, TAC was significantly decreased compared to the start of the study. Compared to 2 years of mobile usage, the levels of total PSA, free PSA, free PSA/total PSA ratio and MDA were increased significantly after 4 years of mobile usage and TAC and serum $\mathrm{Zn}$ were significantly decreased after 4 years compared to 2 years of usage.

\section{Correlation Analyses}

Table 3 shows that, the mobile usage periods per year were significantly negatively correlated with the concentrations of TAC and serum $\mathrm{Zn}$ and significantly correlated with MDA. However, no correlations were detected with the number of mobile devices used or the frequency of daily use.

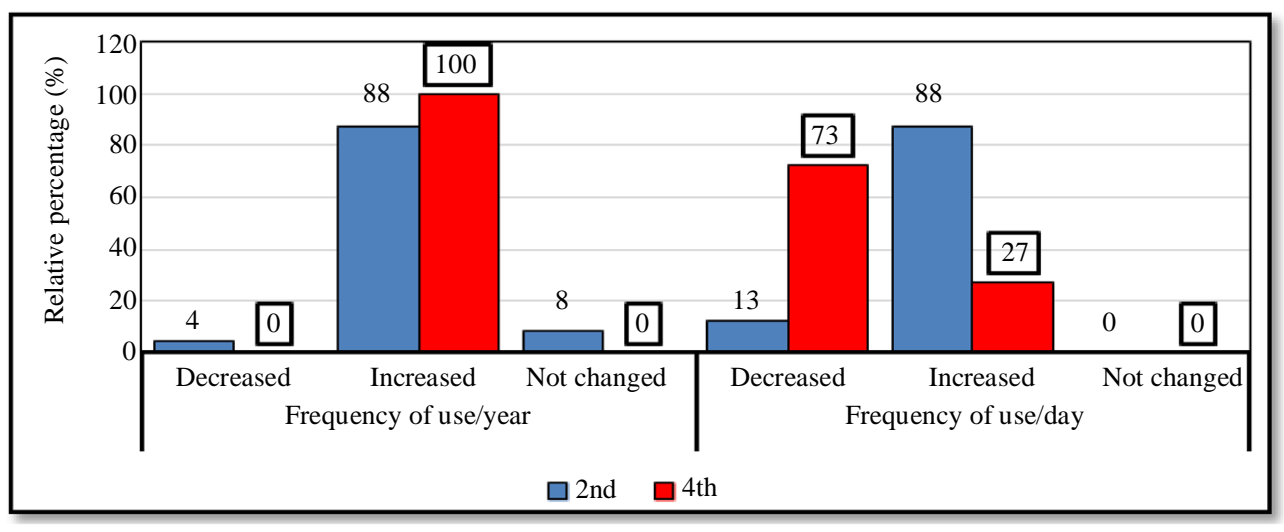

Fig. 1: The change in mobile phone use habits during the 4 years of the study as compared to the beginning of the study 


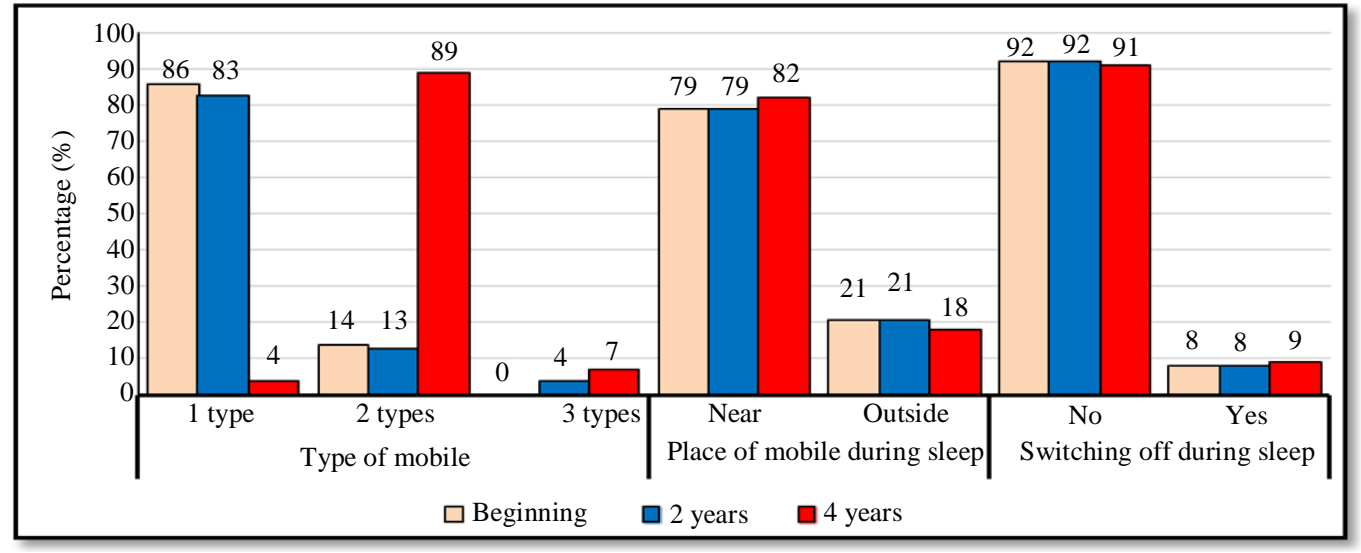

Fig. 2: Differences in the habits of using the mobile phone at the end of the study compared to baseline

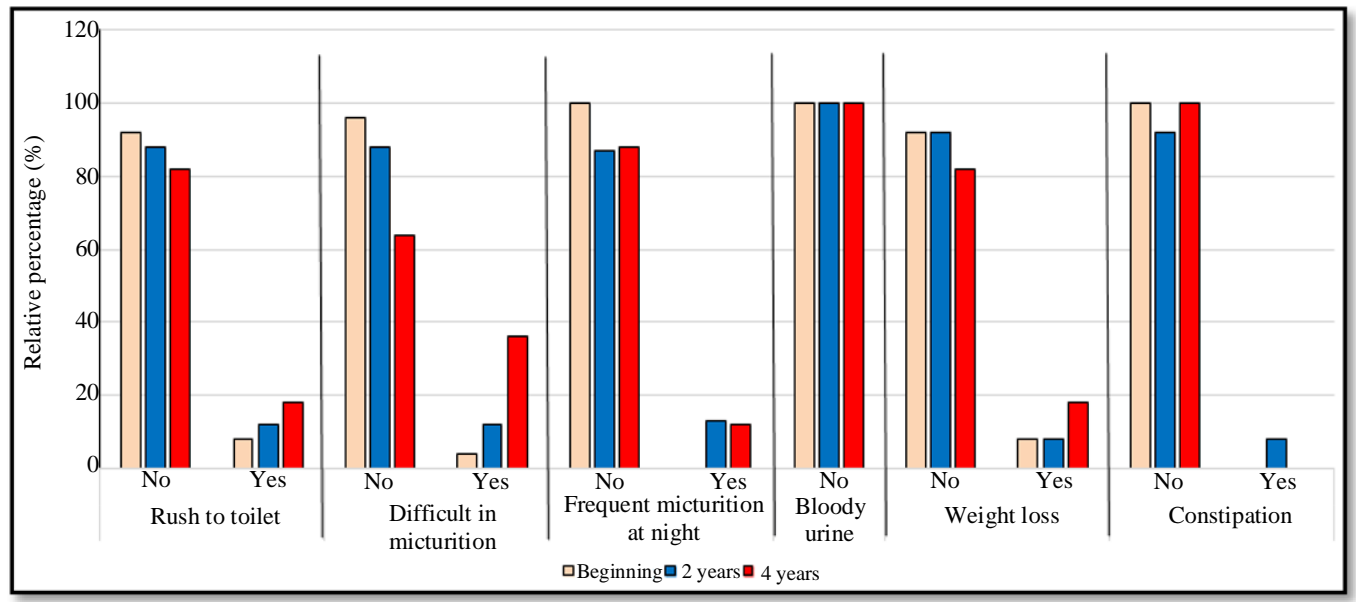

Fig. 3: Changes in the symptoms during the 4 years of using mobile phone

Table 1: Baseline characteristics of the participants

\begin{tabular}{lc}
\hline Number of participants & 28 \\
\hline Age (years) & $30.5 \pm 6.1$ \\
Marital status single/married & $12(43) / 16(57)$ \\
Habits of mobile usage & $18(64) / 10(36)$ \\
Usage period $<10$ years/>10 years & $18(64) / 10(36)$ \\
Usage hour/day <3 h/day/>3 h/day & \\
Phone position during sleeping & $23(82)$ \\
Near the bed & $5(18)$ \\
Outside the room & \\
Mode during sleeping & $26(93)$ \\
Switched off & $2(7)$ \\
Switched on & \\
Complaints & $24(86) / 4(14)$ \\
Rush to the toilet (N/Y) & $24(86) / 4(14)$ \\
Night rush to the toilet (N/Y) & $25(89) / 3(11)$ \\
Difficulty in passing urine (N/Y) & $26(93) / 2(7)$ \\
Constipation (N/Y) & $19(68) / 9(32)$ \\
General pain (N/Y) & $25(89) / 3(11)$ \\
Weight loss (N/Y) &
\end{tabular}


Table 2: Changes in the biochemical parameters through the study period

\begin{tabular}{llll}
\hline Parameter & At the beginning & After 2 years & After 4 years \\
\hline Total PSA $(\mathrm{ng} / \mathrm{ml})$ & $0.50(0.32-0.77)$ & $0.40(0.22-0.61)$ & $0.75(0.67-0.93)^{\mathrm{a}, \mathrm{b}}$ \\
Free PSA $(\mathrm{ng} / \mathrm{ml})$ & $0.17(0.10-0.30)$ & $0.10(0.06-0.14)^{\mathrm{a}}$ & $0.31(0.28-0.39)^{\mathrm{a}} \mathrm{b}$ \\
Free PSA/total PSA ratio & $0.36(0.26-0.50)$ & $0.27(0.14-0.40)^{\mathrm{a}}$ & $0.41(0.40-0.42)^{\mathrm{b}}$ \\
MDA $(\mathrm{nmol} / \mathrm{ml})$ & $8.30(6.1-16.0)$ & $32.50(22.9-57.8)^{\mathrm{a}}$ & $65.00(57.3-75.3)^{\mathrm{a}, \mathrm{b}}$ \\
TAC $(\mathrm{mM})$ & $0.39(0.27-0.43)$ & $0.33(0.22-0.36)^{\mathrm{a}}$ & $0.23(0.20-0.27)^{\mathrm{a}, \mathrm{b}}$ \\
Serum Zinc $(\mu \mathrm{g} / \mathrm{dl})$ & $106.00(91.9-136.8)$ & $73.00(43.2-102.8)^{\mathrm{a}}$ & $97.6(79.2-105.2)$ \\
\hline
\end{tabular}

N.B.: Data are expressed as median (interquartile range)

PSA: Prostate-Specific Antigen, MDA: Malondialdehyde, TAC: Total Antioxidant Capacity

In multiple comparisons, ${ }^{\mathrm{a}} p<0.05 \mathrm{Vs}$ the beginning and ${ }^{\mathrm{b}} p<0.05 \mathrm{Vs}$ two years' follow-up period

Table 3: Correlation analysis of mobile phone usage habits with the studied parameters

\begin{tabular}{|c|c|c|c|c|c|c|}
\hline & \multicolumn{2}{|c|}{$\begin{array}{l}\text { Mobile using period } \\
\text { (years) }\end{array}$} & \multicolumn{2}{|c|}{$\begin{array}{l}\text { Number of mobile } \\
\text { types used }\end{array}$} & \multicolumn{2}{|c|}{$\begin{array}{l}\text { Frequency of } \\
\text { using (hour/day) }\end{array}$} \\
\hline & $\mathrm{r}$ & P-value & $\mathrm{r}$ & P-value & $\mathrm{r}$ & P-value \\
\hline MDA (nmol/ml) & 0.4 & 0.000 & 0.20 & 0.13 & 0.20 & 0.112 \\
\hline TAC (mM/L) & -0.3 & 0.003 & -0.10 & 0.250 & -0.20 & 0.144 \\
\hline $\mathrm{Zn}(\mu \mathrm{g} / \mathrm{dl})$ & -0.4 & 0.032 & 0.02 & 0.907 & 0.06 & 0.757 \\
\hline Total PSA (ng/ml) & 0.2 & 0.231 & 0.20 & 0.431 & 0.20 & 0.311 \\
\hline Free PSA (ng/ml) & 0.1 & 0.346 & 0.20 & 0.460 & 0.10 & 0.612 \\
\hline Free PSA/total PSA ratio & 0.1 & 0.356 & 0.20 & 0.291 & 0.20 & 0.721 \\
\hline
\end{tabular}

Table 4: Correlation analysis of prostatic markers and the oxidative antioxidants parameters

\begin{tabular}{|c|c|c|c|c|c|c|c|c|c|c|c|c|}
\hline & \multicolumn{4}{|c|}{ Beginning } & \multicolumn{4}{|c|}{ 2nd year } & \multicolumn{4}{|c|}{ 4th year } \\
\hline & \multicolumn{2}{|c|}{ TPSA } & \multicolumn{2}{|c|}{ FPSA } & \multicolumn{2}{|c|}{ TPSA } & \multicolumn{2}{|c|}{ FPSA } & \multicolumn{2}{|c|}{ TPSA } & \multicolumn{2}{|c|}{ FPSA } \\
\hline & $\mathrm{r}$ & P-value & $\mathrm{r}$ & P-value & $\mathrm{r}$ & P-value & $\mathrm{r}$ & $\mathrm{P}$-value & $\mathrm{r}$ & P-value & $\mathrm{r}$ & P-value \\
\hline MDA (nmol/ml) & 0.2 & 0.41 & -0.1 & 0.657 & -0.10 & 0.551 & -0.1 & 0.656 & -0.2 & 0.560 & -0.2 & 0.525 \\
\hline TAC (mM/L) & 0.2 & 0.262 & 0.2 & 0.353 & 0.30 & 0.207 & 0.1 & 0.516 & 0.6 & 0.040 & 0.6 & 0.04 \\
\hline $\mathrm{Zn}(\mu \mathrm{g} / \mathrm{dl})$ & 0.1 & 0.559 & -0.1 & 0.701 & 0.03 & 0.908 & -0.1 & 0.714 & 0.2 & 0.472 & 0.3 & 0.425 \\
\hline
\end{tabular}

In Table 4, prostatic markers (TPSA, FPSA and the ratio) were significantly correlated with TAC after 4 years of the study, but no significant correlations were detected between them at the beginning and after 2 years of the study.

\section{Discussion}

Smartphone technology with continuous internet connection leads to an increase in the number of users and intensity of their usage all over the world. In Egypt at the end of the year 2015, the total cell phone subscriptions were around 94 million and reached around 96 million subscriptions by January 2020 (EMCIT, 2015). As people are continuously exposed to RF-EMFs from cell phone use in everyday life, which has been amplified over the years, it is important to note that many males carry cell phones in their trouser pockets. Clipping cell phones to the belts while using hands-free devices exposes the urinogenital organs to a higher power density of the mobile phone radiation than a cell phone would in the 'Standby mode' in a trouser pocket (Jeong et al., 2018). It is therefore important to investigate the effect of long-term use of mobile phones on the prostate.

This study was conducted through four years of follow-up to estimate the effects of prolonged usage of cell phones on the oxidant-antioxidant balance and the deleterious effect on the prostate. There was no significant change in the habits of using the mobile phone, even during sleep, all along the period of the study. However, it was logical that the duration and the frequency of mobile usage per year increased significantly after the second and fourth year of use compared to the start of the study (baseline), while the frequency of usage per day was variated between a significant increase after two years and a significant decrease at the end of the study compared to the beginning of the study.

In this study, after four years of follow-up of the included males, the percent of prostatic complaints were increased compared to that at the beginning of the study but not to the level of significance; especially rushing to the toilet, weight loss and difficulty in urination. Moreover, no one complained of frequent nighttime urination at the beginning of the study, but, 13 and $12 \%$ 
of the males complained after the second and the fourth year, respectively. Symptoms traditionally thought to correlate with PC include Lower Urinary Tract Symptoms (LUTS), such as nocturia and poor urinary stream, erectile dysfunction and visible hematuria (Merriel et al., 2018). Studies have found an increased risk of localized PC in men with LUTS which might be attributed to the higher rates of testing; these patients are mainly diagnosed with early-stage prostate cancer (Merriel et al., 2018; Bhindi et al., 2017).

Furthermore, serum levels of total and free PSA, as well as free PSA/total PSA ratio were significantly increased after a follow-up period of 4 years. Additionally, a significant decrease in the levels of $\mathrm{TAC}$ and $\mathrm{Zn}$ along with a significantly elevation in the MDA levels was observed compared to the beginning of the study. This could be explained by an increase in the stimulation of ROS secondary to EMFs exposure from the daily use of the cell phones during the period of the study. This is consistent with the results obtained by (Höytö et al., 2008) who confirmed that lipid peroxidation is significantly increased following exposure to EMF in L929 fibroblast cells and SH-SY5Y mouse cells.

Awad and Hassan (2008) observed an increase in the oxidative stress biomarker after exposure to cell phones in rats exposed to $900 \mathrm{MHz}$ EMF from cell phones for $1 \mathrm{~h} /$ day during one week. Additionally, (Irmak et al., 2002) detected disturbance in free radical metabolism or induction of oxidative stress by cell phone radiation in some animal studies. They observed that SOD and TAC activities were a significantly decreased in Rabbits animals exposed to EMF. Moreover, (Nasser et al., 2018) proved that in nursing students, there was significant increase in the oxidative stress (MDA) with the increase both duration of mobile ownership and intensity of mobile usage and the oxidantantioxidant status was also significantly affected.

Studies on the health effects of RF energy from communication systems have revealed non-thermal effects that are not directly associated with temperature change but rather to some other changes in the tissues in association with the amount of energy absorbed (Leszczynski et al., 2002; Challis, 2005). Other studies have shown that these effects are mediated by the generation of ROS which can be extremely toxic to cellular homeostasis (Cui et al., 2004; Tkalec et al., 2007). Their cytotoxic effects derive from the peroxidation of membrane phospholipids which creates a change in the conductivity of the membrane and loss of membrane integrity. Exposure to EMF has been observed to cause increased ROS production in the cellular environment. Additionally, the antioxidant defense mechanisms are impaired by being subjected to
EMF that causes overproduction of ROS and thus resulting in oxidative stress (Venugopal et al., 2002).

Moreover, some studies have suggested that EMF promotes free radical activity in cells via another mechanism named the Fenton reaction which is a catalytic process that converts hydrogen peroxide, a product of mitochondrial oxidative respiration, into a highly toxic hydroxyl free radical (Aydin and Akar, 2011; Lai and Singh, 2004).

In the present study, it has been shown that long term use of cell phone causes redox imbalance; as a result of the decrease in TAC and the increase in MDA. Nasser et al. (2018) detected a significant effect of long period of cell phone usage for more than 5 years on the elevation in MDA and decrease in SOD levels compared to that of the users for less than 5 years. Ames et al. (1993; Basaga, 1990) showed that free radicals play a major role in many disease mechanisms, such as cancer and diabetes. Thus, the elevation of oxidative stress in cell phone users could result in an increase the possibility of developing prostatic abnormalities among the male users.

The current study revealed that the mobile usage periods per year were negatively correlated with TAC and serum $\mathrm{Zn}$ level and positively correlated with MDA. However, no correlations were detected with the number of mobile devices used or the frequency of daily use. This could be explained by the evidence that the habits of cell phone usage did not change significantly during the period of the study. As a result, the effect of the use of more than one mobile device could not be studied in the current study due to the short period of exposure to multiple mobile devices, as it was detected in the fourth year of the study. Moreover, it was shown that the frequency of mobile use per day has fluctuated during the study as it increased during the second year and decreased significantly during the fourth year of the study.

Further, PC biomarkers (total PSA, free PSA and their ratio) did not correlate with the period of mobile use, the number of mobile usages, or the frequency of daily use of the cell phones at the beginning as well as during the study. In contrast, prostatic markers were significantly correlated with TAC in the third blood sample (after four years of the study), but no significant correlations were detected between them at the beginning or after 2 years of the study. This could mean that chronic exposure to RF-EMW emitted from the cell phones over a long period of several years has led to the appearance of a significant reduction in TAC which was reflected on the prostatic cancer biomarkers. In addition, the direct thermal harmful effects of the mobile devices on the prostatic cells must not be neglected and further studies are needed. 


\section{Conclusion}

The main purpose of the study was to draw attention to the dangerous effects of cell phones which play a role in the increased oxidative stress-related diseases. The current study pointed out that the long-term use of mobile phones may cause a redox imbalance that is evidenced by a decrease in TAC and an increase in MDA, which might affect eventually the prostate gland as prostate biomarkers were correlated with the level of TAC, which was decreasing with long time of cell phones usage.

\section{Acknowledgment}

The authors would like to thank Shereen Mohamed Abd Elkader at the Department of Clinical and Chemical Pathology, National Cancer Institute, Cairo University for helping to complete tumor marker tests.

\section{Funding Statement}

This research didn't receive any specific grant from funding agencies in the public, commercial, or not for profit sectors.

\section{Authors' Contributions}

Madyha Hassan Mahmoud: Investigation; conducting a research and investigations process and data collection.

Nadia Youssef Morcos: Conceptualization (idea of the research and formulation of overarching research aims)

Khadiga Salah Ibrahim: Methodology; design of the laboratory methodology and supervision of the biochemical analysis.

Amal Saad-Hussein: The corresponding author, design of the study (creation of models), statistical analysis of the results (Formal analysis) and share in editing the manuscript.

Noha Hassan Ibrahim: Validation; verification of the biochemical results.

Ahmed Fathi Soliman: Preparation of the work in form of writing the initial draft.

\section{Ethics}

This article has not been published or presented elsewhere in part or in entirely and is not under consideration by another journal. All the authors have approved the manuscript Authors should address any ethical issues that may arise after the publication of this article.

\section{References}

Agarwal, A., Singh, A., Hamada, A., \& Kesari, K. (2011). Cell phones and male infertility: a review of recent innovations in technology and consequences. International braz j urol, 37(4), 432-454.
Al-Damegh, M. A. (2012). Rat testicular impairment induced by electromagnetic radiation from a conventional cellular telephone and the protective effects of the antioxidants vitamins $\mathrm{C}$ and $\mathrm{E}$. Clinics, 67(7), 785-792.

Ames, B. N., Shigenaga, M. K., \& Hagen, T. M. (1993). Oxidants, antioxidants and the degenerative diseases of aging. Proceedings of the National Academy of Sciences, 90(17), 7915-7922.

Awad, S. M., \& Hassan, N. S. (2008). Health Risks of electromagnetic radiation from mobile phone on brain of rats. J. Appl. Sci. Res, 4(12), 1994-2000.

Aydin, B., \& Akar, A. (2011). Effects of a 900-MHz electromagnetic field on oxidative stress parameters in rat lymphoid organs, polymorphonuclear leukocytes and plasma. Archives of medical research, 42(4), 261-267.

Baah, E., Obirikorang, C., Asmah, R. H., Acheampong, E., Anto, E. O., Yakass, M. B., \& Mawusi, D. (2019). Seminal Antioxidant Capacity to Oxidative Stress Induced by Electromagnetic Waves Emitting from Cellular Phones on Sperm Quality: An in Vitro Simulation Model. Advances in Reproductive Sciences, 7(4), 94-105.

Basaga, H. S. (1990). Biochemical aspects of free radicals. Biochemistry and Cell Biology, 68(7-8), 989-998.

Bhindi, A., Bhindi, B., Kulkarni, G. S., Hamilton, R. J., Toi, A., van der Kwast, T. H., ... \& Fleshner, N. E. (2017). Modern-day prostate cancer is not meaningfully associated with lower urinary tract symptoms: analysis of a propensity score-matched cohort. Canadian Urological Association Journal, 11(1-2), 41.

Challis, L. J. (2005). Mechanisms for interaction between RF fields and biological tissue. Bioelectromagnetics, 26(S7), S98-S106.

Cui, K., Luo, X., Xu, K., \& Murthy, M. V. (2004). Role of oxidative stress in neurodegeneration: recent developments in assay methods for oxidative stress and nutraceutical antioxidants. Progress in NeuroPsychopharmacology and Biological Psychiatry, 28(5), 771-799.

Dasdag, S., \& Akdag, M. Z. (2016). The link between radiofrequencies emitted from wireless technologies and oxidative stress. Journal of chemical neuroanatomy, 75, 85-93.

Desai, K. M., Chang, T., Wang, H., Banigesh, A., Dhar, A., Liu, J., ... \& Wu, L. (2010). Oxidative stress and aging: is methylglyoxal the hidden enemy? Canadian journal of physiology and pharmacology, 88(3), 273-284.

Donkena, K. V., Young, C. Y., \& Tindall, D. J. (2010). Oxidative stress and DNA methylation in prostate cancer. Obstetrics and gynecology international, 2010. 
EMCIT (MCIT) (2015). Egyptian Ministry of Communications and Information Technology http://www.mcit.gov.eg/

Höytö, A., Luukkonen, J., Juutilainen, J., \& Naarala, J. (2008). Proliferation, oxidative stress and cell death in cells exposed to $872 \mathrm{MHz}$ radiofrequency radiation and oxidants. Radiation research, 170(2), 235-243.

Irmak, M. K., Fadıllığlu, E., Güleç, M., Erdoğan, H., Yağmurca, M., \& Akyol, Ö. (2002). Effects of electromagnetic radiation from a cellular telephone on the oxidant and antioxidant levels in rabbits. Cell Biochemistry and Function: Cellular biochemistry and its modulation by active agents or disease, 20(4), 279-283.

Jemal, A., Bray, F., Center, M. M., Ferlay, J., Ward, E., \& Forman, D. (2011). Global cancer statistics. CA: a cancer journal for clinicians, 61(2), 69-90.

Jeong, Y. J., Son, Y., Han, N. K., Choi, H. D., Pack, J. K., Kim, N., ... \& Lee, H. J. (2018). Impact of long-term RF-EMF on oxidative stress and neuroinflammation in aging brains of C57BL/6 mice. International journal of molecular sciences, 19(7), 2103.

Johnsen, Ø., \& Eliasson, R. (1987). Evaluation of a commercially available kit for the colorimetric determination of zinc in human seminal plasma. International Journal of Andrology, 10(2), 435-440.

Kei, S. (1978). Serum lipid peroxide in cerebrovascular disorders determined by a new colorimetric method. Clinica chimica acta, 90(1), 37-43.

Koracevic, D., Koracevic, G., Djordjevic, V. Andrejevic, S., \& Cosic, V. (2001). Method for the measurement of antioxidant activity in human fluids. Journal of clinical pathology, 54(5), 356-361.

Lai, H., \& Singh, N. P. (2004). Magnetic-field-induced DNA strand breaks in brain cells of the rat. Environmental health perspectives, 112(6), 687-694.
Leszczynski, D., Joenväärä, S., Reivinen, J., \& Kuokka, R. (2002). Non-thermal activation of the hsp27/p38MAPK stress pathway by mobile phone radiation in human endothelial cells: molecular mechanism for cancer-and blood-brain barrierrelated effects. Differentiation, 70(2-3), 120-129.

Meena, R., Kumari, K., Kumar, J., Rajamani, P., Verma, H. N., \& Kesari, K. K. (2014). Therapeutic approaches of melatonin in microwave radiationsinduced oxidative stress-mediated toxicity on male fertility pattern of Wistar rats. Electromagnetic Biology and Medicine, 33(2), 81-91.

Merriel, S. W., Funston, G., \& Hamilton, W. (2018). Prostate cancer in primary care. Advances in therapy, 35(9), 1285-1294.

Nasser, S., Amer, N. M., Ghobashi, M. M., Morcos, G., Hafez, S. F., Shaheen, W., \& Helmy, M. A. (2018). Knowledge, Attitude and Practices (KAP) Study and Antioxidant Status among Mobile Phone Users. Bioscience Research, 15(4), 3645-3651.

Parsanezhad, M. E., Mortazavi, S. M., Doohandeh, T., Namavar Jahromi, B., Mozdarani, H., Zarei, A., .. \& Haghani, M. (2015). Exposure to radiofrequency radiation emitted from mobile phone jammers adversely affects the quality of human sperm. International Journal of Radiation Research, 15(1), 63-70.

Tkalec, M., Malarić, K., \& Pevalek-Kozlina, B. (2007). Exposure to radiofrequency radiation induces oxidative stress in duckweed Lemna minor L. Science of the Total Environment, 388(1-3), 78-89.

Venugopal, S. K., Devaraj, S., Yang, T., \& Jialal, I. (2002). $\alpha$-Tocopherol decreases superoxide anion release in human monocytes under hyperglycemic conditions via inhibition of protein kinase $\mathrm{C}-\alpha$. Diabetes, 51(10), 3049-3054. 